\title{
The influence of glucocorticoid receptor SNPs on outcome after haematopoietic stem cell transplantation
}

Jean Norden ${ }^{1}$, Kim F Pearce ${ }^{1}$, Julie A. E Irving ${ }^{2}$, Matthew P. Collin ${ }^{1}$, Xiao N. Wang ${ }^{1}$, Daniel Wolff ${ }^{3}$, Hans-Jochem Kolb ${ }^{4}$, Gerard Socie ${ }^{5}$, Zoya Kuzmina ${ }^{6}$, Hildegard Greinix ${ }^{7}$, Ernst Holler ${ }^{3}$, Vanderson Rocha $^{8}$, Eliane Gluckman ${ }^{8}$, Ilona Hromadnikova ${ }^{9}$, Anne M. Dickinson ${ }^{1}$

${ }^{1}$ Haematological Sciences, Institute of Cellular Medicine, University of Newcastle upon Tyne, UK,

${ }^{2}$ Wolfson Childhood Cancer Research Centre, Northern Institute for Cancer Research, University of Newcastle upon Tyne, UK,

${ }^{3}$ Department of Haematology and Oncology, University of Regensburg, Germany;

${ }^{4}$ Klinikum Grosshadern, III Medical Klinik, Munich, Germany,

${ }^{5}$ Department of Haematology, St Louis Hospital, Paris, France

${ }^{6}$ Department of Internal Medicine I, Bone Marrow Transplantation, Medical University of Vienna, Vienna, Austria,

${ }^{7}$ Department of Internal Medicine, Division of Haematology, Medical University of Graz, Graz, Austria',

${ }^{8}$ EUROCORD, St Louis Hospital, Paris, France,

${ }^{9}$ Department of Molecular Biology and Cell Pathology, Third faculty of Medicine, Charles University, Prague, Czech Republic.

\section{Address for Correspondence:}

Prof. Anne Mary Dickinson

Haematological Sciences

Institute of Cellular Medicine

Newcastle University

Newcastle upon Tyne

NE2 $4 \mathrm{HH}$

United Kingdom

Tel: +44 (0) 1912226794

E-mail: anne.dickinson@newcastle.ac.uk 


\section{Summary}

Haematopoietic stem cell transplantation (HSCT) remains the only cure for most haematological malignancies, however, the mortality rate remains high. Complications after HSCT include relapse, graft versus host disease (GvHD), graft rejection and infection. Over the last few years several groups, have demonstrated that non-HLA gene polymorphisms can be predictive of outcome after HSCT. Since the glucocorticoid cortisol is pivotal in the regulation of the immune system, we decided to examine single nucleotide polymorphisms (SNPs) (rs6198, rs33388 and rs33389) within the glucocorticoid receptor (GR) and correlate with HSCT outcome.

The training set consisted of patients $(n=458)$ who underwent HSCT for acute leukaemia between 1983 and 2005. In the recipients, the absence of the ACT haplotype and absence of the T allele of rs33388 were associated with decreased OS and the absence of the ACT haplotype, the absence of the T allele of rs33388 and the presence of the ATA haplotype were associated with increased risk of relapse. In addition, the presence of the ACT haplotype in the recipient showed a trend to be associated with increased risk of cGvHD. The patients in this cohort received mainly myeloablative conditioning ( $n=327)$. The SNPs in the glucocorticoid receptor were then investigated in a validation set $(n=251)$ of HSCT patients transplanted for acute leukaemia from 2006. This cohort contained significantly more patients that had received reduced intensity conditioning (RIC). Some of the results could be validated in these patients. However, contrary to the training set, the absence of the haplotype ACT in the donor in this cohort was associated with increased risk of cGvHD. Differences in the conditioning were shown to influence the results.

These results are the first to associate GR SNPs with HSCT outcome and demonstrate the inherent problems of replicating SNP association studies in HSCT, due to different pre-transplant regimens.

Key words: Polymorphisms, haplotype, inflammation aGVHD, cGvHD, relapse. 


\section{$\underline{\text { Introduction }}$}

Glucocorticoids such as cortisol are involved in the regulation of the immune system and inflammation, Rhen and Cidowski, (2005) and exert their effect through binding to the glucocorticoid receptor (GR), (HUGO gene nomenclature NR3C1), a transcription factor expressed in most cells. This receptor binds glucocorticoids in the cytoplasm and translocates them to the nucleus, where they influence the expression of many genes, chiefly by interfering with the binding of transcription factor activating protein and nuclear factor $\kappa \beta$ to inflammatory immune genes such as interleukin (IL) IL-1, IL-2, IL-3, IL-6, IL-8, and TNF $\alpha$ and causing their down regulation, Chikanza et al (2003). Glucocorticoids can also induce the transcription of anti-inflammatory genes including IL-4, IL-10, IL13 and transforming growth factor $\beta$ (TGF $\beta$ ), Chikanza et al. (2003).

The human glucocorticoid receptor gene contains 10 exons and has 2 main isoforms, GR- $\alpha$ and GR- $\beta$, which are generated as a result of alternative splicing, Castro et al. (1996), Encio and DeteraWadleigh (1991) ) as can be seen in figure 1. Both isoforms share exons 1 to 8; for the GR- $\alpha$ isoform, exons 1 to $9 \alpha$ are transcribed into GR $\alpha$ mRNA, the functional GR receptor. In the case of the GR$\beta$ isoform, exon 9a is replaced with exon 9b, resulting in GR- $\beta$ mRNA. GR- $\beta$ does not itself bind hormone, but is thought to have an antagonistic effect on GR- $\alpha$ signalling by heterodimerisation with GR- $\alpha$, Castro et al. (1996), Encio and Detera-Wadleigh (1991). Associations have been reported between GR- $\beta$ over expression and autoimmune disease and inflammation, Derijk et al. (2001), Chatzikyriakidou et al. (2009) and Van den Akker et al. (2008) but it is not clear whether this is actually a cause or an effect and whether this increase in GR- $\beta$ is enough to have an effect on the activity of GR- $\alpha$.

The single nucleotide polymorphism (SNP) rs6198 is situated in the $3^{\prime}$ UTR of exon $9 \beta$ of the GR gene and changes a consensus sequence from ATTTA to GTTTA. The ATTTA sequence is known to destabilise GR- $\beta m R N A$, Derijk et al. (2001). The $A$ to $G$ substitution therefore increases the stability of the GR- $\beta$ mRNA, so this $G$ allele is thought to be the GC resistant allele, as more GR- $\beta$ is available to bind to GR- $\alpha$ which in turn gives less cortisol induced immunosuppression. The $G$ allele of rs6198 has been associated with rheumatoid arthritis (RA), Derijk et al. (2001), Chatzikyriakidou et al. (2009), heart disease, Van den Akker et al. (2008) and reduced nasal carriage of staphylococcus aureus, Van den Akker et al. (2006). More recently, a trend to an association has been found with the $\mathrm{G}$ allele of rs6198 in the patient and corticosteroid sensitive aGvHD, O'Meara et al (2015).

In addition, SNPs in intron B of the GR gene have been shown to predispose to RA susceptibility, Chatzikyriakidou et al. (2009). The SNPs rs33388 (A to T substitution) and rs33389 (C to Tsubstitution) are in intron $B$ and are in partial linkage disequilibrium.Carriage of the variant T allele of rs33388 has been positively associated with the incidence of RA ( $p=0.025)$. The AT haplotype of rs33388 and rs33389 has also been associated with increased glucocorticoid sensitivity, Stevens et al. (2004) and it exhibits higher sensitivity to steroid treatment in children with nephritic syndrome, Zalewski et al. (2008). These SNPs are thought to influence gene transcription by altering consensus recognition sites for SR proteins, Cartegni (2002), which are serine arginine rich factors involved in alternative splicing of RNA. Indeed, interrogation of the splicing factor map (SFMAP) database suggests that rs33388 lies in consensus recognition sites for the RNA splicing factors, QK1 (rs33388) and SRp20, also known as SRSF3 (rs33389) and, in fact, the T allele of rs33389 is predicted to create 
additional QK1 and SRp20 sites. In addition, rs33388 and rs33389 have roles not only in mRNA splicing, but also in export of mRNA from the nucleus and in protein translation, Paz et al. (2010).

We hypothesised that SNPs in the GR gene may be associated with outcome after HSCT and influence acute and chronic GVHD, relapse, NRM and OS. We therefore examined the role of SNPs rs6198, rs33389 and rs33388 and their haplotypes on NRM, OS, relapse and the incidence of acute and chronic GVHD in a (training) cohort of 458 adult patient/donor pairs undergoing HSCT for acute leukaemia pre-2006. The role of these SNPs was then further evaluated in a second smaller (validation) cohort of 251 adult patient and donor pairs undergoing HSCT for acute leukaemia post 2005.

\section{Materials and Methods}

\section{Patients}

The training set consisted of 458 adult HLA matched sibling and HLA matched unrelated patient and donor pairs from eight European transplant centres, where $66 \%$ had received T cell replete stem cell grafts. They were analysed retrospectively and all of the patients had undergone HSCT for acute leukaemia. Transplants were conducted between November 1983 and December 2005. Relapse was defined as haematological relapse. The main causes of death were relapse (46\%), infection (21\%) and GvHD (18\%). The validation cohort consisted of 251 adult HLA matched sibling and matched unrelated patient and donor pairs undergoing HSCT for acute leukaemia from January 2006 to January 2010 at four European centres. This cohort was also analysed retrospectively and the main causes of death were relapse (51.2\%), GvHD (25\%) and infection (14.3\%). There was no overlap of patients between the cohorts. The samples from the patients and donors were obtained following informed consent and approval in accordance with EBMT guidelines and the diagnosis of GVHD was made following the standard criteria, Glucksberg et al. (1974). The majority of the cohort after the year 2000 had high-resolution tissue typing for HLA Class I A,B,C and Class II DP,DQ and DR.

\section{Genotyping}

DNA was prepared from archived frozen peripheral blood mononuclear cells by standard techniques, consisting of SDS lysis, proteinase K digestion, phenol chloroform extraction followed by ethanol precipitation. Genotyping for the SNPs rs6198, rs33388 and rs33389 was outsourced to Kbioscience (http://www.kbioscience.co.uk) using their own fluorescence based competitive PCR technology (KASPar). They also designed the assays for the SNPs based on the DNA sequence (50 bases) on either side of the SNP (Figure 1).

\section{Statistical Analysis}

The impact of the selected GR SNPs on survival was assessed via Kaplan Meier (log rank) tests and Cox regression modelling. NRM and relapse were analyzed using the method of competing risks, Kaplan Meier (log rank) tests and Cox regression modelling. As clinical data were categorical, differences between cohorts were assessed using the chi-square test with exact $p$ values; the Cochran Armitage test for trend was also utilized for ordinal data. Due to the lack of time-to-event data for acute GvHD and chronic GvHD, statistical associations were assessed via chi-square based statistics and binary logistic regression modelling. Effect sizes (including hazard ratios (HRs), odds 
ratios (ORs) and Cramér's V) together with their 95\% confidence intervals (Cls) are reported. Univariable associations were initially evaluated; the potential confounding effect of transplant date was then assessed via multivariable modelling were appropriate. Significance is reported at the $5 \%$ level. SPSS (version 23.0) and R (version 3.1.2) were used for the computations.

\section{$\underline{\text { RESULTS }}$}

\section{Clinical Characteristics}

Table 1 describes patient characteristics for the two cohorts. Transplants 2006 onwards statistically showed more patients were over 40 years of age, a lower proportion of female donor to male patient transplants, more HLA-matched unrelated donors, more reduced intensity conditioning (RIC) protocols, more peripheral blood stem cell transplants, less NRM, and less acute GvHD grade II-IV. Median follow-up times were 77 months and 19 months in the training and validation sets respectively.

\section{GR SNP Frequencies in Our Cohorts}

The occurrence of the variant alleles was comparable to that for published Caucasian allele frequencies for rs33388 and rs33389, but significantly different for rs6198 (all allele frequencies taken from the National centre for biotechnology information) as shown in Table 2. This difference could be because the published cohorts were actually smaller than in our study cohort, indeed as can be seen from table 2 for r 6198 the allele frequency in our cohort falls between the two published allele frequencies for Caucasians. The SNPs are in Hardy-Weinberg equilibrium ( $p=>0.05)$ and partial linkage disequilibrium Donn et al. (2007) and therefore, we were also able to assign each patient or donor a specific haplotype (i.e. ACT, ATA, ACA and GCA and the SNP order for the haplotype designation is rs6198, then rs33389, then rs33388).

\section{$\underline{\text { Training Set }}$}

None of the GR SNP variables were found to be associated with NRM. None of the donor GR variables were found to be associated with OS. In the recipient, absence of the ACT haplotype ( $p=0.013, \mathrm{HR}: 0.665,95 \% \mathrm{Cl}: 0.477-0.926)$ and absence of the T allele of $r 333388$ ( $p=0.041$, HR: 0.727 , 95\% Cl: 0.531-0.995) were associated with decreased OS. The Kaplan Meier survival curves for these SNPs are shown in Figures 2a and $2 b$.

None of the donor GR SNP variables were found to be associated with relapse. The absence of the ACT haplotype, the presence of the haplotype ATA and the absence of the T allele of rs33388 in the recipient were found to be associated with increased risk of relapse $(p=0.007, \mathrm{HR}: 0.539,95 \% \mathrm{Cl}$ : 0.340-0.854; $p=0.037$, HR: 1.597, 95\% Cl: $1.019-2.505$ and $p=0.004$, HR: $0.543,95 \% \mathrm{Cl}: 0.354-0.831$ respectively) (Table 3 ). The cumulative incidence plots for relapse for the recipient GR haplotype ACT and ATA, and the recipient rs33388 any T allele are shown in Figure $3 a \mathrm{ab}$ and $3 \mathrm{c}$. 
None of the recipient or donor GR SNP variables were found to be associated with the incidence or severity of acute GvHD. For CGvHD, there was an indication that carriage of the ACT haplotype in the recipient was associated with the incidence of chronic GvHD ( $p=0.075$, OR: $1.833,95 \% \mathrm{Cl}$ : 0.966 3.478).

\section{Validation Set}

In this cohort, none of the GR SNP variables in either patients or donors were associated with NRM and OS. As in the training set, none of the donor GR SNP variables were found to be associated with relapse; in the recipient, in contrast to the training set, the presence of the ACA haplotype was associated with an increased risk of relapse ( $p=0.042$, HR: $1.921,95 \% \mathrm{Cl}: 1.003-3.678)$ (Table 3$)$, the cumulative incidence plots are shown in Figure $3 \mathrm{~d}$.

Further differences were observed between the training and validation sets with regard to acute and chronic GvHD.

In contrast to the training set, the presence of the ACT haplotype in the recipient was associated with more severe aGvHD (grades II-IV) ( $p=0.009$, OR: 4.073, 95\% CI: 1.337-12.410) and the absence of the $A$ allele of rs33388 was also associated with more severe aGvHD ( $p=0.037, O R: 0.420,95 \% \mathrm{Cl}$ : 0.190-0.929) as shown in Table 4. In addition, in the donor, the absence of the ATA haplotype and the absence of the T allele of rs33389 was associated with the occurrence of aGvHD (grades I-IV) ( $p=0.001$, OR: $0.285,95 \% \mathrm{Cl}: 0.133-0.607$ for both genetic factors) as can be seen in Table 4 , and both also showed a trend to be associated with more severe grades of aGvHD (II-IV) ( $p=0.055$, OR: $0.422,95 \% \mathrm{Cl}: 0.184-0.965$ for both genetic factors). Interestingly we found that the absence of the ACT haplotype in the donor was significantly associated with cGvHD ( $p=0.025,0 R: 0.374,95 \% \mathrm{Cl}$ : 0.163-0.857) as shown in Table 4.

\section{$\underline{\text { Myeloablative and RIC Cohorts }}$}

The two cohorts were then combined and split into two new cohorts based on the type of pretransplant conditioning, either RIC or myeloablative, and the analysis repeated. Again none of the SNP variables in the donor or recipient were associated with NRM in either of these cohorts. In the myeloablative cohort, the new finding was that the presence of the $\mathrm{G}$ allele of rs6198 in the recipient was associated with reduced OS ( $p=0.046$, HR: $1.401,95 \% \mathrm{Cl}: 1.001-1.964)$ ). For relapse, the absence of the T allele of rs33388 and the absence of the ACT haplotype in the recipient, as shown previously in the training cohort, was associated with increased incidence ( $p=0.028, H R: 0.603,95 \%$ $\mathrm{Cl}: 0.380-0.957, \mathrm{p}=0.048, \mathrm{HR}: 0.603,95 \% \mathrm{Cl}: 0.362-0.999$ respectively). In addition, the presence of the GCA haplotype and the presence of the $\mathrm{G}$ allele of rs6198 in the recipient were associated with increased risk of relapse (0.028, HR: $1.703,95 \% \mathrm{Cl}: 1.047-2.768$ and $\mathrm{p}=0.018, \mathrm{HR}: 1.741,95 \% \mathrm{Cl}$ :

1.087-2.788 respectively). None of the SNP variables were found to be associated with aGvHD but, as in the trend observed in the training set, the presence of the ACT haplotype in the recipient was associated with the incidence of $\mathrm{cGvHD}$ ( $p=0.046$, OR: $2.012,95 \% \mathrm{Cl}: 1.044-3.880)$.

In the RIC cohort, the presence of the ACT haplotype and the presence of the T allele of rs33388 in the donor was associated with reduced OS ( $p=0.02, \mathrm{HR}: 1.711,95 \% \mathrm{Cl}: 1.070-2.735$ and $\mathrm{p}=0.025, \mathrm{HR}$ : $1.659,95 \% \mathrm{Cl}: 1.051-2.619$, respectively). The absence of the $\mathrm{G}$ allele of rs6198 in the recipient was associated with increased risk of relapse ( $p=0.048$, HR: $0.510,95 \% \mathrm{Cl}: 0.256-0.999)$. The (double) 
presence of the ACT haplotype, the absence of the A allele of rs33388 and the absence of the T allele of rs33389 in the recipient was associated with more severe aGvHD (II-IV) ( $p=0.021$, OR: $2.598,95 \%$ $\mathrm{Cl}: 1.152-5.860 ; \mathrm{p}=, 0.037$; OR: $0.420,95 \% \mathrm{Cl}: 0.190-0.931$ and $\mathrm{p}=0.049, \mathrm{OR}: 0.497,95 \% \mathrm{Cl}: 0.252-$ 0.982 , respectively) and also, in the donor, the absence of the ATA haplotype and the absence of the T allele of $r 33389$ was associated with the incidence of aGvHD ( $p=0.03$, OR: $0.464,95 \%$ Cl: 0.241 0.893 and $p=0.024$, OR: $0.474,95 \% \mathrm{Cl}: 0.250-0.900$, respectively). No GCR SNP associations were found for CGVHD in this cohort.

As the training and validation sets have been amalgamated, it may be argued that year of transplant could act as a confounder in these analyses, thus the relationship between each SNP and outcome was again assessed via Cox regression or logistic regression (as appropriate) after controlling for time period (1983-2005 vs 2006-2010). As depicted in Table 5, results are virtually unchanged after adjustment

The ACT haplotype was associated with GvHD in the patient in the training, validation, myeloablative and RIC cohorts. In the training set and the myeloablative set it was associated with the risk of cGvHD ( $p=0.075$, OR: 1.833, 95\% Cl: 0.966-3.478, and p=0.046, OR: 2.012, 95\% Cl: 1.044-3.880, respectively) and in the validation and RIC set, it was significantly associated with severe aGvHD (grades II-IV) ( $p=0.009$, OR: $4.073,95 \% \mathrm{Cl}: 1.337-12.410$ and $p=0.021$, OR: $2.598,95 \% \mathrm{Cl}$ : $1.152-$ 5.860 , respectively). In addition, the absence of the A allele of rs33388 in both the validation and RIC sets was significantly associated with severe aGvHD ( $p=0.037$, OR: $0.420,95 \% \mathrm{Cl}: 0.190-0.929$ and 0.037, OR: 0.420, 95\% Cl: 0.190-0.931, respectively).

\section{Discussion}

HSCT remains the main curative therapy for patients with acute leukaemia, however the mortality rate remains high (40 to 60\%), Bensinger and Storb (2001). In recent years several groups, including ourselves, have reported that non-HLA gene polymorphisms are predictive of outcome in HSCT, Dickinson et al. (2004). We therefore decided to investigate SNPs in the GR gene, as they have been shown to be involved in the inflammatory process and autoimmune disease, Balavarca et al (2015), Pearce et al (2016).

We did not find any associations of the SNPs rs6198, rs33388 and r33389 with NRM in either the recipient or the donor in both cohorts in all analyses. In the first cohort, we found absence of the ACT haplotype ( $p=0.013$, HR: $0.665,95 \% \mathrm{Cl}: 0.477-0.926)$ and the absence of the T allele of $r s 3388$ ( $p=0.041, \mathrm{HR}: 0.727,95 \% \mathrm{Cl}: 0.531-0.995)$ in the recipient was associated with decreased OS. The ACT haplotype contains two GC resistant alleles; the C allele of rs33389 and the T allele of rs33388 so it could be that carriage of this inflammatory haplotype might be aiding the graft versus leukaemia (GVL) effect and therefore, the absence of this haplotype would result in a more immunosuppressed phenotype giving rise to reduced OS. Indeed, when the incidence of relapse was investigated we found that for the first cohort in the recipient, the absence of the haplotype ACT, the presence of the haplotype ATA and the absence of the T allele of $\mathrm{rs} 33388$ was significantly associated with increased incidence of relapse ( $p=0.007, \mathrm{HR}: 0.539,95 \% \mathrm{Cl}: 0.340-0.854 ;, p=0.037, \mathrm{HR}: 1.597,95 \% \mathrm{Cl}$ : 1.019-2.505 and $\mathrm{p}=0.004, \mathrm{HR}: 0.543,95 \% \mathrm{Cl}: 0.354-0.831$, respectively). These variants could therefore be influencing the GVL effect. In fact, in the second cohort, we found that the presence of the ACA haplotype in the recipient was associated with an increased risk of relapse $(p=0.042, H R$ : 
1.921, 95\% Cl: 1.003-3.678). This haplotype could also be detrimental to the GVL effect as, like the ATA haplotype, this haplotype does not contain the inflammatory T allele of rs33388.

We found no significant associations in either the recipient or the donor for the incidence of acute GvHD in the first cohort. However, in the second cohort, we found that the presence of the ACT steroid resistant haplotype and the absence of the A allele of rs33388 in the recipient was associated with more severe aGvHD (grades II-IV) ( $p=0.009$, OR: $4.073,95 \% \mathrm{Cl}: 1.337-12.410$ and $p=0.037, \mathrm{OR}$ : $0.420,95 \% \mathrm{Cl}: 0.190-0.929$ respectively). We also found in the donor the absence of the ATA haplotype was associated with the occurrence of aGvHD (grades I-IV) ( $p=0.001$, OR: $0.285,95 \% \mathrm{Cl}$ : 0.133-0.607) and showed a trend to be associated with more severe grades (II-IV). In addition, in the donor, the absence of the T allele of 33389 was associated with the occurrence of aGvHD (grades IIV) ( $p=0.001$, OR: $0.285,95 \% \mathrm{Cl}: 0.133-0.607)$ and showed a trend to be associated with more severe grades (II-IV). This could be due to the absence of the inflammatory or GC resistant T allele of rs33388 in the ATA haplotype. Indeed, in the first cohort, we found that the presence of the ATA haplotype associated with relapse ( $p=0.037, \mathrm{HR}: 1.597,95 \% \mathrm{Cl}: 1.019-2.505)$. Previous studies have shown the T allele of rs33388 to be associated with RA, Chatzikyriakidou et al. (2009) and the TA haplotype of rs33389 and rs33388 to be associated with GC sensitivity, Stevens et al. (2004). In the first cohort, the presence of the GC resistant ACT haplotype in the recipient showed a trend to an association with cGvHD ( $p=0.075)$. Conversely, in the second cohort, we found that the absence of this resistant haplotype in the donor was significantly associated with the incidence of cGvHD ( $p=0.025$, OR: $0.374,95 \% \mathrm{Cl}: 0.163-0.857$ ), this could be due to the biology of the transplant

Transplant protocols have also changed dramatically over the last twenty years. In our second cohort the number of transplants using matched unrelated donors, transplants using peripheral blood stem cells as the source of stem cells and the use of RIC protocols were significantly higher. The instigation of RIC protocols for older patients and patients with co-morbidities, Slavin et al. (1998) could account for our results. Since the patients in the first cohort received chiefly myeloablative conditioning and more of those in the second cohort received reduced intensity conditioning, we decided to combine the first and second cohort, divide it into myeloablative and RIC groups and reanalyse. In addition, we adjusted for transplant period (1983-2005 vs 2006-2010) and the results were compared with those of the unadjusted analysis (leading to virtually identical conclusions).

For the cohort that received myeloablative conditioning $(n=457)$ the presence of the $G$ allele of rs6198 in the recipient was associated with reduced OS and increased risk of relapse $(p=0.046, H R$ : 1.401, 95\% Cl: $1.001-1.964$ and $p=0.018, H R: 1.741,95 \% \mathrm{Cl}: 1.087-2.788$ respectively). The presence of the GCA haplotype in the recipient was also associated with increased risk of relapse $(p=0.028$, HR: $1.703,95 \% \mathrm{Cl}: 1.047-2.768)$. Interestingly, a recent study investigating the influence of SNPS in the GCR in steroid sensitive and steroid resistant aGvHD found the presence of the $G$ allele of rs6198 in the recipient showed a trend for association with steroid sensitive aGvHD, O'Meara et al (2015) indicating that in a HSCT setting the G allele is the steroid sensitive allele. The presence of the ACT haplotype in the recipient was associated with cGvHD ( $p=0.046$, OR: $2.012,95 \% \mathrm{Cl}: 1.044-3.880$ ), in the first cohort a trend for this association was also observed. These results strengthen our hypothesis that the presence of SNPs which incur GC resistance induce more cGvHD and hence more GVL following myeloablative conditioning. There is a well-documented association between the occurrence of cGvHD and reduced relapse risk in HSCT, Signori et al. (2012) so this could explain our findings in the first patient cohort and the reanalysed cohort which received myeloablative 
conditioning. In patients undergoing myeloablative conditioning therapy, carriage of the T allele rs33388 could exert its effect in the recipient in the first phase (cytokine storm) of GvHD/GvL induction, Nestel et al. (1992). Interestingly in both the first cohort and myeloablative conditioning cohort, none of the variables were significant for incidence and severity of aGvHD.

Conversely for the RIC cohort ( $n=252)$, the presence of the T allele of rs33388 and the ACT haplotype in the donor were found to be associated with reduced OS, ( $p=0.025, \mathrm{HR}: 1.659,95 \% \mathrm{Cl}: 1.051-2.619$ and $0.02, \mathrm{HR}: 1.711,95 \% \mathrm{Cl}: 1.070-2.735$ respectively) and the absence of the $\mathrm{G}$ allele of rs6198 in the recipient was associated with increased risk of relapse ( $p=0.048$, HR: $0.510,95 \% \mathrm{Cl}: 0.256-0.999)$. In this cohort we found the absence of the A allele of rs33388, the absence of the T allele of rs 33389 and the (double) presence of the ACT haplotype significantly associated with severe aGvHD (grades II=IV), ( $p=0.037$, OR: $0.420,95 \% \mathrm{Cl}: 0.190-0.931 ; p=0.049$, OR: $0.497,95 \% \mathrm{Cl}: 0.252-0.982$ and $\mathrm{p}=0.021$, OR: $2.598,95 \% \mathrm{Cl}: 1.152-5.860$, respectively). In addition, the absence of the ATA haplotype and the absence of the T allele of rs33389 in the donor was associated with the incidence of aGvHD (grades I-IV) ( $p=0.03$, OR: $0.464,95 \% \mathrm{Cl}: 0.241-0.893$ and $p=0.024$, OR: $0.474,95 \% \mathrm{Cl}$ : 0.250-0.900). No associations were found for CGvHD in this cohort. Interestingly, for patients having RIC for HSCT, the conditioning regimen that initiates the first phase of GVHD/GvL, induction is less inflammatory, van der Velden et al. (2010). However, in both the second and RIC cohorts similar "inflammatory" genetic variables were significant for the incidence of aGvHD. The presence of the ACT haplotype and the absence of the A allele of rs33388 in the recipient were associated with severe aGvHD. In addition, in both of these cohorts, the absence of the ATA haplotype and the absence of the T allele of rs33389 in the donor were associated with the incidence of aGvHD.

This is the first study to show that SNPs in the GR gene are associated with outcome post HSCT, and in addition this study also highlights the effects that different transplant regimens can have on SNP association studies.

\section{Disclosures}

Ethics

REC reference 14/NE/1136, IRAS project ID 129780.

\section{Acknowledgements}

This work was funded by CELLEUROPE, FP7 Marie Curie Initial Training Network (Contract No: 315963).

The authors declare no conflict of interests. 


\section{$\underline{\text { References }}$}

Balavarca Y., Pearce K.F., Norden J., Collin M., Jackson G., Holler E... Bickeböller H. (2015). Predicting survival using clinical risk scores and non-HLA immunogenetics. Bone Marrow Transplantation. 50, 1445-1452. doi: 10.1038/bmt.2015.

Bensinger, W.I. \& Storb, R. (2001) Allogeneic peripheral blood stem cell transplantation. Rev Clinical Experimental Hematology, 5(2), 67-86.

Cartegni, L., Chew, S.L. \& Krainer, A.R. (2002). Listening to the silence and understanding nonsense: exonic mutations that effect splicing. Nature Reviews Genetics, 3, 285-298. doi: $10.1038 / \mathrm{nrg} 775$

De Castro, M., Elliot, S., Kino, T., Bamberger, C., Karl, M., Webster, E. \& Chrousos, G.P. (1996).The non-ligand binding B-isoform of the human glucocorticoid receptor: tissue level, mechanisms of action and potential physiologic role Molecular Medicine, 2, 597-607.

Chatzikyriakidou, A., Georgiou, I., Voulgari, P.V., Georgiadis, A.N., Argyriou, E.S. \& Drosos, A.A. (2009) Glucocorticoid receptor variants may dispose to rheumatoid arthritis susceptibility. Scandanavian Journal of Rheumatology, 38, 1-5. doi: 10.1080/03009740802366068.

Chikanza, I.C., Kozaci, D. \& Chernajovsky, Y. (2003) The molecular and cellular basis of corticosteroid resistance. Journal of Endocrinology, 179, 301-310.

Derijk, R.H., Scaaf, M.J., Turner, G., Datson, N.A., Vreugdenhil, E., Cidlowski, J... DeteraWadleigh, S.D. (2001) A human glucocorticoid receptor gene variant that increases the stability of the glucocorticoid receptor $\beta$-isoform mRNA is associated with rheumatoid arthritis. Journal of Rheumatology, 28, 2283-2288.

Dickinson, A.M., Middleton, P.G., Rocha, V., Gluckman, E. \& Holler, E. (2004) Genetic polymorphisms predicting the outcome of bone marrow transplants. British Journal of Haematology, 127, 479-490. doi: 10.1111/j.1365-2141.2004.05216.x

Donn, R., Payne, D. \& Ray, D. (2007) Glucocorticoid gene receptor polymorphisms and susceptibility to rheumatoid arthritis. Clinical Endocrinology, 67,342-345. doi: 10.1111/j.1365-2265.2007.02887.x

Encio, I.J. \& Detera-Wadleigh, S.D. (1991) The genomic structure of the human glucocorticoid receptor. Journal of Biological Chemistry, 266, 7182-7188.

Glucksberg, H., Storb, R., Fefer, A. Buckner, C.D., Neiman, P.E,, Clift, R.A., Lerner, K.G. \& Thomas, E.D.(1974) Clinical manifestations of acute graft versus host disease in human recipients of marrow from HLA matched sibling donors. Transplantation, 18(4),295-304.

Nestel, F.P., Price, K.S., Seemayer, T.A. \& Lapp, W.S. (1992) Macrophage priming and lipopolysaccharide-triggered release of tumour necrosis factor alpha during graft-versushost disease. Journal of Experimental Medicine, 175, 405-413. 
O'Meara, A., Boukouaci, W., Robin, M., Xhaard, A., Fortier, C., Marzais, F...Tamouza, R. (2015) GLCCI1 and Glucocorticoid Receptor Genetic Diversity and Response to Glucocorticoid-Based Treatment of Graft-versus-Host Disease. Biology of Blood and Marrow Transplantation,21(7):1246-1250. doi: 10.1016/j.bbmt.2015.03.015

Paz, I., Akerman, M., Dror, I., Kosti, I \& Mandel-Gutfreund, Y. (2010) SFmap: a web server for motif analysis and prediction of splicing factor binding sites. Nucleic Acids Research, 38(Web Server issue): W281-5. 18.

Pearce K.F., Balavarca Y., Norden J., Jackson G., Holler E., Dressel R... Dickinson A.M. (2016) Impact of genomic risk factors on survival after hematopoietic stem cell transplantation for patients with acute leukemia. International Journal of Immunogenetics 43, 404-412. doi: 10.1111/iji.12295

Reddy, P. (2003) Pathophysiology of acute graft versus host disease. Hematology Oncology, 21(4),149-161. doi: 10.1002/hon.716

Rhen, T. \& Cidlowski, J.A. (2005) Anti-inflammatory actions of glucocorticoids-new mechanisms for old drugs. New England Journal of Medicine, 353(16),1711-1723. doi: 10.1056/NEJMra050541

Signori, A., Crocchioli, R., Oneto, R., Sacchi, N., Sormani, M.P., Fagioli, F... Bacigalupo, A. (2012) Chronic GVHD is associated with lower relapse risk irrespective of stem cell source among patients receiving transplantation from unrelated donors. Bone Marrow Transplantation, 47, 1474-1478. doi: 10.1038/bmt.2012.58

Slavin, S., Nagler, A., Naparstek, E. Kapelushnik, Y., Aker, M., \& Cividalli, G. (1998) Nonmyeloablative stem cell transplantation and cell therapy as an alternative to conventional bone marrow transplantation with lethal cytoreduction for the treatment of malignant and nonmalignant haematologic diseases. Blood, 91, 756-763.

Stevens, A., Ray, D.W., Zeggini, E., John, S., Richards, H.L., Griffiths, C.E. \& Donn, R.(2004) Glucocorticoid sensitivity is determined by a specific glucocorticoid receptor haplotype. Journal of Clinical Endocrinology and Metabolism, 89, 892-897. doi: 10.1210/jc.2003-031235

Van den Akker, E.L., Nouwen, J.L., Melles, D.C., van Rossum, E.F., Koper, J.W., Uitterlinden, A.G. et al. (2006). Staphylococcal aureus nasal carriage is associated with glucocorticoid receptor polymorphisms. Journal of Infectious Diseases, 194, 814-818. doi: 10.1086/506367

Van den Akker, E.L., Koper, J.W., Van Rossum, E.F.C., Dekker, M.J.H., Russcher, H., de Jong, F.H. et al. (2008) The glucocorticoid receptor gene and the risk of cardiovascular disease. Archives Internal Medicine, 68, 33-39. doi: 10.1001/archinternmed.2007.41

Van der Velden, W.J., Herbers, A.H., Feuth, T., Schaap, N.P., Donnelly, J.P., Blijlevens, N.M. (2010) Intestinal damage determines the inflammatory response and early complications in patients receiving conditioning for a stem cell transplantation. PLoS One. 20;5(12):e15156. doi: 10.1371/journal.pone.0015156. 
Zalewski, G., Wasilewska, A., Zoch-Zwierz, W. \& Chyczewski, L. (2008) Response to prednisone in relation to NR3C1 intron B polymorphisms in childhood nephritic syndrome. Paediatric Nephrology, 2008, 1073-1078. doi: 10.1007/s00467-008-0772-7. 
Legend for Figure 1 Structure of the glucocorticoid gene, exons are numbered and the introns are labelled with letters. The positions of the SNPs that were investigated are marked with the putative GC resistant allele shown in red and the order of the SNPS in our haplotypes in green.

Legend for Figure 2 Kaplan Meier survival curves for GR SNPs in the first cohort. a) Survival curves of the GR haplotype ACT in the recipient, log rank test $p=0.013, \mathrm{HR}: 0.665,95 \% \mathrm{Cl}: 0.477-0.926 . \mathrm{b})$ Survival curves for the T allele of GR 33388 in the recipient, log rank test $p=0.041, \mathrm{HR}: 0.727,95 \% \mathrm{Cl}$ : 0.531-0.995. In all graphs the black line corresponds to absence of the haplotype/allele and the grey line corresponds to presence. Crosses represent censored observations.

Legend for Figure 3 Cumulative incidence of relapse a) For GR haplotype ACT in the first cohort, the black line corresponds to the absence of the ACT haplotype in the recipient and the grey line its presence (Gray's test, $p=0.026$, cumulative incidence at 2 years for ACT present: $0.245,95 \% \mathrm{Cl}$ : 0.187-0.308; cumulative incidence at 2 years for ACT absent: $0.400,95 \% \mathrm{Cl}: 0.284-0.512$ ). b) For GR haplotype ATA in the first cohort, the black line corresponds to the absence of the ATA haplotype in the recipient and the grey line its presence (Gray's test, $p=0.037$, cumulative incidence at 2 years for ATA present: $0.378,95 \% \mathrm{Cl}: 0.272-0.483$; cumulative incidence at 2 years for ATA absent: 0.246 ,95\% Cl: 0.186-0.311). c) For GR SNP rs33388 allele T in the first cohort, the black line corresponds to absence of T allele in the recipient and grey line corresponds to its presence (Gray's test, $p=0.011$, cumulative incidence at 2 years for allele T present: $0.248,95 \% \mathrm{Cl}: 0.191-0.309$; cumulative incidence at 2 years for allele T absent: $0.402,95 \% \mathrm{Cl}$ : 0.295-0.505). d) For GR haplotype ACA in the second cohort, the black line corresponds to the absence of the ACA haplotype in the recipient and the grey line its presence (Gray's test, $p=0.037$, cumulative incidence at 2 years for ACA present: $0.363,95 \% \mathrm{Cl}: 0.232-0.496$; cumulative incidence at 2 years for ACA absent: $0.222,95 \% \mathrm{Cl}: 0.127$ $0.332)$. 
Table 1. Patient characteristics for the training $(N=458)$ and validation $(N=251)$ sets.

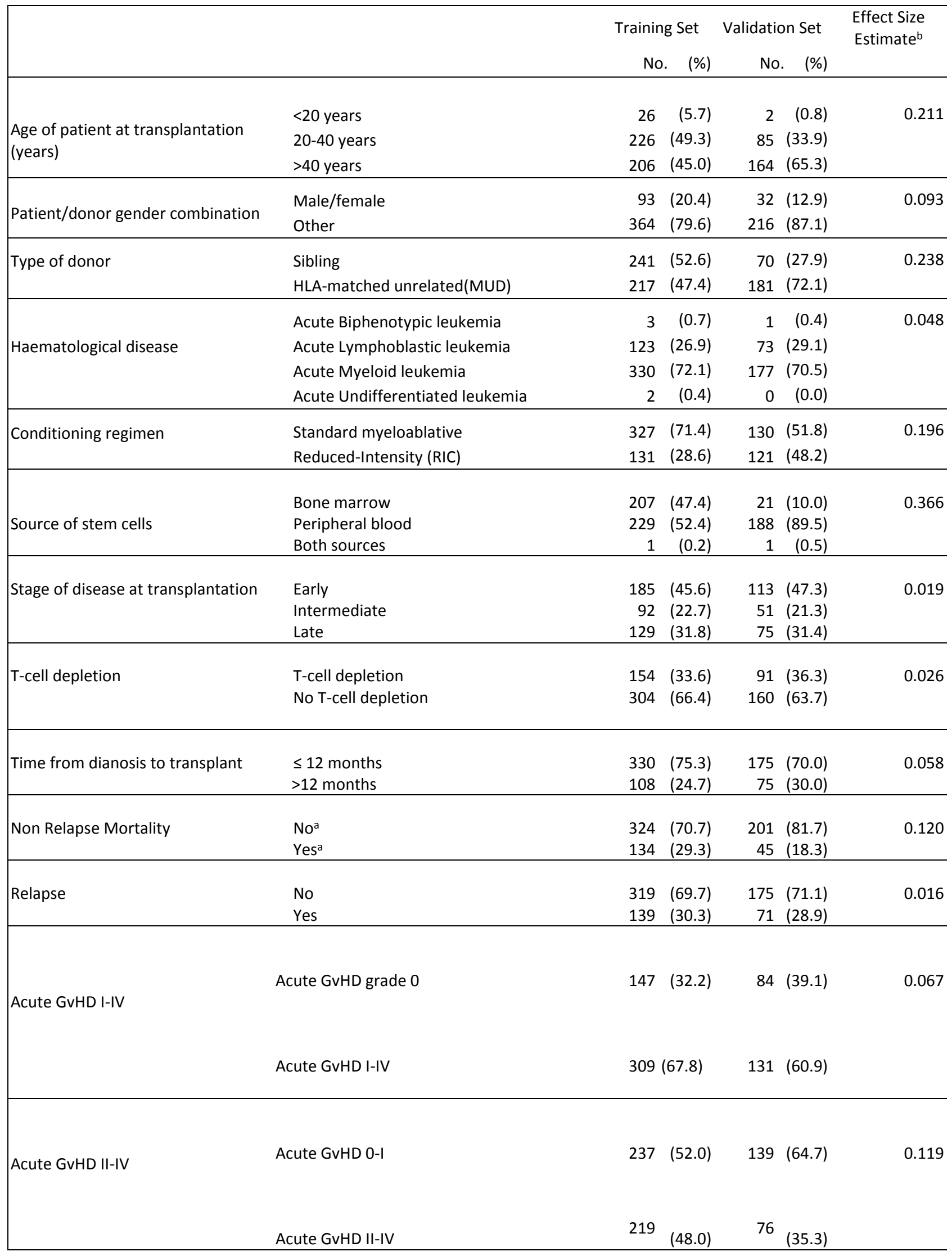


Acute GvHD 0-II

Acute GvHD III-IV

No chronic GvHD

Chronic GvHD
368

$(80.7)$

$171(79.5)$

0.014

$88 \quad(19.3)$

$44(20.5)$

Chronic GvHD

155

(46.1) 76

(48.4)

0.021

181

(53.9)

81

(51.6)

Abbreviation : GvHD= graft versus host disease

aNon-relapse mortality (NRM) = 'Yes' defined as patient dead without ever experiencing a prior relapse; non-relapse mortality (NRM) = 'No' defined as patient alive without ever experiencing a prior relapse or patient has had a relapse.

b Cramér's V with a range from 0 to 1 . For this set of results, Cramér's V statistics of $0.1,0.3$ and 0.5 are considered small, medium and large effect sizes respectively (Cohen, 1988). 
Table 2 GR SNP Frequencies in Our Cohort

\begin{tabular}{|l|l|l|}
\hline & $\begin{array}{l}\text { Published } \\
\text { Caucasian } \\
\text { frequency }\end{array}$ & $\begin{array}{l}\text { Frequency in our } \\
\text { population }\end{array}$ \\
\hline$\underline{\mathrm{rs} 6198}$ & $0.73 \quad 0.90$ & 0.841 \\
\hline $\mathrm{A}$ & $0.27 \quad 0.10$ & 0.159 \\
\hline $\mathrm{G}$ & 0.549 & 0.542 \\
\hline$\underline{\mathrm{rs33388}}$ & 0.451 & 0.458 \\
\hline $\mathrm{A}$ & & 0.839 \\
\hline $\mathrm{T}$ & 0.858 & 0.161 \\
\hline$\underline{\mathrm{rs33389}}$ & 0.142 & \\
\hline C & & \\
\hline T & & \\
\hline
\end{tabular}


Table 3

a) Significant associations between relapse and the presence/absence of GR SNP variables in the training set for the recipient.

\begin{tabular}{|l|l|l|l|l|}
\hline Variable & $\begin{array}{l}\text { Log rank test } \\
\text { (p value) }\end{array}$ & Hazard Ratio & $\begin{array}{l}\text { Confidence } \\
\text { Interval (95\%) }\end{array}$ & $\begin{array}{l}\text { Level associated } \\
\text { with Relapse }\end{array}$ \\
\hline ATA haplotype & 0.037 & $1.597^{\mathrm{a}}$ & $1.019-2.505$ & Presence \\
\hline ACT haplotype & 0.007 & $0.539^{\mathrm{b}}$ & $0.340-0.854$ & Absence \\
\hline rs33388 T allele & 0.004 & $0.543^{\mathrm{c}}$ & $0.354-0.831$ & Absence \\
\hline
\end{tabular}

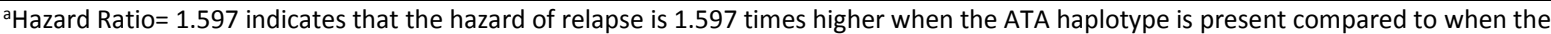
ATA haplotype is absent; ${ }^{\text {bHazard Ratio }} \mathbf{0 . 5 3 9}$ indicates the hazard of relapse is lower when the ACT haplotype is present compared to when the ACT haplotype is absent (0.539 times the hazard); 'Hazard Ratio= 0.543 indicates the hazard of relapse is lower when the rs33388 T allele is present compared to when the rs33388 T allele is absent ( 0.543 times the hazard)

b) Significant associations between relapse and the presence of the ACA haplotype in the recipient GCR in the validation set.

\begin{tabular}{|l|l|l|l|l|}
\hline Variable & $\begin{array}{l}\text { Log rank test } \\
\text { ( } p \text { value) }\end{array}$ & Hazard Ratio ${ }^{a}$ & $\begin{array}{l}\text { Confidence } \\
\text { Interval (95\%) }\end{array}$ & $\begin{array}{l}\text { Level associated } \\
\text { with Relapse }\end{array}$ \\
\hline ACA haplotype & 0.042 & 1.921 & $1.003-3.678$ & Presence \\
\hline
\end{tabular}

${ }^{\text {a Hazard Ratio }} 1.92$ indicates that the hazard of relapse is 1.92 times higher when the ACA haplotype is present compared to when the ACA haplotype is absent 
Table 4 Associations between aGvHD and cGvHD and presence/absence of GR SNP variables in the validation cohort

\begin{tabular}{|l|l|l|l|l|}
\hline Variable & $\begin{array}{l}\text { Chi- } \\
\text { square } \\
\text { test } \\
\text { (p-value) }\end{array}$ & Odds Ratio & $\begin{array}{l}\text { Confidence } \\
\text { Interval (95\%) }\end{array}$ & $\begin{array}{l}\text { Level associated } \\
\text { with GvHD }\end{array}$ \\
\hline Recipient ACT haplotype $^{\mathrm{b}}$ & 0.009 & 4.073 & $1.337-12.410$ & Presence \\
\hline Recipient rs333888 A allele $^{\mathrm{b}}$ & 0.037 & 0.420 & $0.190-0.929$ & Absence \\
\hline Donor ATA haplotype & 0.001 & 0.285 & $0.133-0.607$ & Absence \\
\hline Donor rs333889 T allele $^{c}$ & 0.001 & 0.285 & $0.133-0.607$ & Absence \\
\hline Donor ACT haplotype $^{\mathrm{d}}$ & 0.025 & 0.374 & $0.163-0.857$ & Absence \\
\hline
\end{tabular}

alnterpretation: Odds Ratio $=4.073$ indicates that the odds of aGvHD II-IV is 4.073 times higher when the ACT haplotype is present compared to when the ACT haplotype is absent; Odds Ratio $=0.420$ indicates the odds of aGvHD II-IV is lower when the rs333888 A allele is present compared to when the $\mathrm{r} 333888 \mathrm{~A}$ allele is absent ( 0.420 times the odds) etc.

${ }^{\mathrm{b}}$ Association with aGvHD grades II-IV.

'Association with aGvHD grades I-IV,

${ }^{\mathrm{d} A s s o c i a t i o n}$ with $\mathrm{CGvHD}$.

Abbreviation: $\mathrm{aGvHD}=$ acute graft vs host disease, $\mathrm{CGvHD}=$ chronic graft versus host disease 
Table 5 Associations between aGvHD and cGvHD and presence/absence of GR SNP variables adjusted and non-adjusted analysis comparison

\begin{tabular}{|c|c|c|}
\hline & Unadjusted association with outcome & Adjusted association with outcome $\mathrm{a}^{\mathrm{a}}$ \\
\hline & Odds Ratio/Hazard ratio $(95 \% \mathrm{Cl})$ & Odds Ratio/Hazard ratio $(95 \% \mathrm{Cl})$ \\
\hline \multicolumn{3}{|l|}{ Myeloablative } \\
\hline \multicolumn{3}{|l|}{ Overall Survival } \\
\hline Recipient rs6198 G allele & $1.401(1.001-1.964)$ & $1.402(1.001-1.966)$ \\
\hline \multicolumn{3}{|l|}{ Relapse } \\
\hline Recipient rs33388 T allele & $0.603(0.380-0.957)$ & $0.608(0.382-0.968)$ \\
\hline Recipient ACT haplotype & $0.603(0.362-0.999)$ & $0.608(0.364-0.999)$ \\
\hline Recipient GCA haplotype & $1.703(1.047-2.768)$ & $1.709(1.051-2.778)$ \\
\hline Recipient rs6198 G allele & $1.741(1.087-2.788)$ & $1.746(1.091-2.797)$ \\
\hline \multicolumn{3}{|l|}{$c G v H D$} \\
\hline Recipient ACT haplotype & $2.012(1.044-3.880)$ & $1.998(1.031-3.871)$ \\
\hline \multicolumn{3}{|l|}{$\begin{array}{l}\text { Reduced Intensity } \\
\text { Conditioning }\end{array}$} \\
\hline \multicolumn{3}{|l|}{ Overall Survival } \\
\hline Donor rs33388 T allele & $1.659(1.051-2.619)$ & $1.673(1.058-2.648)$ \\
\hline Donor ACT haplotype & $1.711(1.070-2.735)$ & $1.713(1.070-2.742)$ \\
\hline \multicolumn{3}{|l|}{ Relapse } \\
\hline Recipient rs6198 G allele & $0.510(0.256-0.999)$ & $0.510(0.256-0.999)$ \\
\hline \multicolumn{3}{|l|}{$a G v H D \| I-I V$} \\
\hline $\begin{array}{l}\text { Recipient ACT ACT } \\
\text { haplotype }\end{array}$ & $2.598(1.152-5.860)$ & $2.524(1.104-5.771)$ \\
\hline Recipient rs33388 A allele & $0.420(0.190-0.931)$ & $0.434(0.193-0.975)$ \\
\hline Recipient rs33389 T allele & $0.497(0.252-0.982)$ & $0.466(0.232-0.935)$ \\
\hline$a G v H D I-I V$ & & \\
\hline
\end{tabular}




\begin{tabular}{|l|l|l|}
\hline Donor ATA haplotype & $0.464(0.241-0.893)$ & $0.462(0.240-0.891)$ \\
\hline Donor rs33389 T allele & $0.474(0.250-0.900)$ & $0.473(0.249-0.899)$ \\
\hline
\end{tabular}

adjusted for time period (1983-2005 vs 2006-2010)

Abbreviation: $\mathrm{aGvHD}=$ acute graft vs host disease, $\mathrm{cGvHD}=$ chronic graft versus host disease 